Int. J. Morphol.,

33(3):842-849, 2015.

\title{
Valoración Clínica del Riesgo, Interpretación y Utilidad Práctica
}

\author{
Clinical Risk Assessment, Interpretation and Practical Utility
}

\author{
Carlos Manterola D..$^{*, *, * * *, * * * * *}$ \& Tamara Otzen H. ${ }^{* * *, * * * * *, * * * * * * * *}$
}

MANTEROLA, D. C. \& OTZEN, H. T. Valoración clínica del riesgo, interpretación y utilidad práctica. Int. J. Morphol., 33(3):842$849,2015$.

RESUMEN: Los clínicos que observan la evidencia para una mejor atención de sus pacientes, deberían familiarizarse no sólo con algunas herramientas de lectura crítica de la literatura biomédica, sino que también con algunos conceptos epidemiológicos y herramientas estadísticas de utilidad que permiten valorar la validez de estos estudios. Nos referimos de forma especial a las medidas de asociación y de impacto que permiten resumir información estadística recogida de los artículos en cuestión, a modo de resumen de datos y análisis e interpretación de resultados, tanto de significación estadística como clínica. Entendemos que esto no es fácil ya que se trata de conceptos áridos y a veces incomprensibles; sino que porque además, no es común que el personal sanitario tenga incorporado el razonamiento probabilístico; y porque finalmente, la manera en que se informan este tipo de resultados no es la más didáctica que pudiese ser. Sin embargo, dada la progresiva proliferación y cada vez mayor disponibilidad de publicaciones científicas, se hace cada vez más imprescindible conocer y entender estos términos y su utilidad en la práctica clínica. Es por ello, que el objetivo de este manuscrito es describir los conceptos relacionados con la valoración del riesgo, conocer la modalidad de cálculo de las principales medidas de asociación y de impacto; así como también, su interpretación y aplicación práctica.

PALABRAS CLAVE: Riesgo, Incidencia; Estudio de cohorte; Ensayo clínico; Medidas de asociación; Epidemiología clínica; Medicina basada en evidencia.

\section{INTRODUCCIÓN}

Es propio de los estudios clínicos que las relaciones causales propuestas entre las variables se traduzcan en términos de probabilidad. Por ende, se trata de establecer si la probabilidad de ocurrencia de un fenómeno observado se debe a los factores que se sospecha que intervienen en su génesis y no al azar. Para lograr este objetivo, cualquier investigación en salud debe medir la frecuencia de uno o varios eventos de interés y realizar comparaciones entre los diferentes grupos que se estudian, o en el mismo grupo a través del tiempo (Moreno-Altamirano et al., 2000; Pineda Ovalle et al., 2006).

La investigación debe determinar también, si la presencia de otros factores (exposición a un probable agente causal o intervención), pueden modificar la frecuencia del evento estudiado. Eso significa que debe concluir de una forma válida, si existe una asociación causal entre los factores de riesgo (FR) o de intervención y un evento de interés (EI). Suponiendo que las conclusiones de la investigación sean válidas, la utilidad de las mismas dependerá de la relevancia o el impacto que éstas tengan al aplicarlas en pacientes diferentes a los de la investigación, por ejemplo, en nuestros pacientes. Por esta razón, el clínico debería interpretar las diferentes medidas que se expresan en las publicaciones científicas para tomar decisiones basadas en la validez de estas en el tratamiento de sus pacientes (Pineda Ovalle et al.; Manterola \& Cortés, 2010).

Sin embargo la interpretación de los resultados puede resultar más complicada, ya que, si bien el procedimiento para evaluar la magnitud del efecto de un tratamiento está muy bien establecido (MacMahon\& Trichopoulos, 1996;

* Departamento de Cirugía y Traumatología, Universidad de La Frontera, Temuco, Chile.

** Centro de Excelencia en Estudios Morfológicos y Quirúrgicos (CEMyQ), Universidad de La Frontera, Temuco, Chile.

*** Programa de Doctorado en Ciencias Médicas, Universidad de La Frontera, Temuco, Chile.

***** Centro de Investigación en Biociencias, Universidad Autónoma de Chile, Temuco, Chile.

****** Escuela de Psicología, Universidad Autónoma de Chile, Temuco, Chile.

******* Fellow Researcher, Universidad Científica del Sur, Lima, Perú. 
Fletcher et al., 2003; Manterola \& Cortés), la manera de expresarlo no lo está tanto; lo que se genera en parte debido a la existencia de diversas formas (todas válidas y correctas), de expresar este efecto (Abraira, 2000).

Es conocido el hecho, que la percepción de los clínicos sobre la magnitud del efecto de un tratamiento, depende de la forma en que éste se exprese (Palmeiro Fernández et al., 2004; Fahey, 1995). Así, dependiendo de la medida de efecto que decida utilizar el autor, el impacto de una intervención podrá aparecer de mayor o menor magnitud (Replogle \& Johnson, 2007).

Por lo tanto, parece razonable familiarizarse con las distintas medidas de asociación y efecto existentes y sus relaciones; toda vez que éstas son utilizadas con la finalidad de establecer asociaciones y eventuales relaciones causales; y por ende, de proponer alternativas para la prevención y control de problemas de salud.

El objetivo de este manuscrito es describir los conceptos relacionados con la valoración del riesgo, conocer la modalidad de cálculo de las principales medidas de asociación y de impacto; así como también, su interpretación y aplicación práctica.

\section{CONCEPTO DE RIESGO EN EPIDEMIOLOGÍA}

El concepto de riesgo es probabilístico; y se refiere a la probabilidad de ocurrencia de un evento o fenómeno en una población o grupo, perteneciente a un lugar determinado y durante un período de tiempo determinado. El evento o fenómeno puede ser una enfermedad o un EI.

Suele expresarse mediante una variable dicotómica (sí/no; ocurre/no ocurre). Por ejemplo recidiva del cáncer, ocurrencia de un infarto, muerte, curación, etc. Por otra parte, si se trata de variables continuas, se pueden categorizar (niveles de colesterol y triglicéridos; o tensión arterial). Por ejemplo hipercolesterolemia, hipertensión o hipotensión arterial.

La manera apropiada de presentarlo es a través del resultado de una proporción que se puede graficar como (sujetos con el EI / grupo de sujetos en riesgo de presentar el EI). Expresado de otra forma individuos de una población presentan un evento (nE) y el número total de individuos de la población susceptible de presentarlo (nP).

Por otra parte, cualquier tipo de probabilidad se expresa siempre por un número que va de 0 a 1 , pero en medicina se ha adoptado referir al riesgo en términos de porcentaje; de tal modo que esto representa a cúantos individuos de cada 100 padecerán el evento. Conceptualmente, éste es el riesgo absoluto de la población y corresponde a la incidencia (Figs. 1A y 1B, respectivamente).

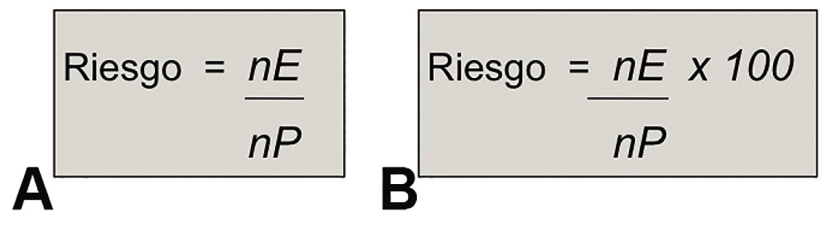

Fig. 1. Fórmulas para el cálculo del riesgo bruto (A) y en porcentaje (B).

Desde el punto de vista de la epidemiología clínica, no todos los individuos tienen la misma probabilidad de enfermar o de desarrollar un EI; el que varía en función de la presencia o ausencia de características asociadas como edad, sexo, peso, hábitos, etc. Por lo tanto, un FR es "una característica individual o colectiva, endógena o exógena, asociada al aumento de la incidencia de una enfermedad o EI en una población, a partir de la posibilidad de aparición de este EI en el individuo" (Akobeng, 2005). Estos FR (biológicos, ambientales, de comportamiento, socio-culturales o económicos) pueden, sumándose unos a otros, modificar el efecto aislado de cada uno de ellos y producir un fenómeno de interacción (Pita Fernández et al., 2002).

\section{MEDICIONES DE LA FRECUENCIA DE UNA EN- FERMEDAD O EI}

Antes de abocarnos de lleno al riesgo, nos parece conveniente dedicar algunas líneas a conceptos relacionados que sin duda permiten comprender mejor lo que viene a continuación.

En primer término, es oportuno aclarar que la ocurrencia de enfermedades o EI, puede ser medida a través de tasas y proporciones; entendiendo como Tasa a una razón como una proporción con especificación de tiempo, que nos dice que tan rápido ocurre un determinado EI en una población (por ejemplo [muertes en 2013 / población en 2013] x 1000). Por otra parte, entendemos como proporción a una razón en la que el numerador está incluido en el denominador, por lo cual, nos habla de que proporción de la población es afectada por el EI en estudio (por ejemplo hombres / total de nacimientos).

Las tasas pueden ser utilizadas para estimar riesgo si el periodo de tiempo es corto (anual) y la incidencia de la enfermedad o EI en este periodo es relativamente constante. Las Tasas pueden ser crudas, específicas y ajustadas. Las tasas crudas representan el número de eventos en una población en un periodo de tiempo determinado (año calenda- 
rio), por lo que suelen reflejar la probabilidad de un evento (por ejemplo: Como la probabilidad de muerte aumenta con la edad, la tasa cruda de mortalidad refleja la estructura de edad de la población). Por su parte, las tasas específicas se utilizan para construir tasas para segmentos específicos de la población de manera de comparar entre diferentes grupos o estratos (edad, raza, género, niveles socioculturales, etc.). $\mathrm{Y}$, las tasas ajustadas se usan para comparar tasas en población general, ajustando por aquellas variables que podrían influir en los resultados (edad, género, raza, causas de mortalidad, etc.).

Una de las tasas más utilizadas es la "tasa de incidencia", que corresponde al número de eventos durante un periodo de tiempo a los que está expuesta una población en riesgo ([ $\mathrm{N}^{\mathrm{o}}$ eventos en tiempo / población en riesgo] x 1000).

Por otra parte, el riesgo expresa en general la probabilidad de un resultado adverso; en unidades que van desde el 0 al 1 (sin riesgo a riesgo de $100 \%$ ); requiere de un periodo de referencia y refleja la incidencia acumulada de una enfermedad o EI en ese periodo de tiempo.

El estudio de riesgo está basado en el cálculo de la frecuencia de la enfermedad o EI. En el ámbito de la epidemiología clínica, la frecuencia de un EI se traduce en dos mediciones bien definidas: prevalencia e incidencia.

La prevalencia alude al número de casos de una enfermedad o EI en un lugar y tiempo determinados. Es un indicador de existencia o de "stock", ya que considera todos los casos presentes, sean nuevos o antiguos. Es particularmente útil para medir la existencia de enfermedades crónicas en la población. Los estudios utilizados para determinar prevalencia son los estudios de corte transversal (Manterola \& Otzen, 2014). Una prevalencia alta puede reflejar un riesgo alto o una supervivencia prolongada sin cura; y una prevalencia baja puede representar bajo riesgo, progresión rápida y fatal de la enfermedad, o cura rápida.

Por ejemplo, el cáncer de páncreas es de baja incidencia, corta duración y baja prevalencia. La diabetes mellitus es de baja incidencia, larga duración y alta prevalencia. Por su parte, la hipertensión arterial es de alta incidencia, larga duración y alta prevalencia.

Por otro lado, la incidencia, corresponde al número de casos nuevos del EI, que se presentan durante un período de seguimiento. Por ende, este indicador incorpora un concepto de temporalidad a la determinación, aludiendo al riesgo de desarrollar el EI en un periodo de tiempo determinado. Se trata entonces de un indicador de flujo, da cuenta de la velocidad con que aparecen nuevos casos (puede ser ex- presada como número absoluto o como tasa). Los estudios utilizados para determinar incidencia son los estudios de cohorte (Manterola \& Otzen).

De esta forma, y según el objetivo del análisis, se pueden extraer entre otros, los siguientes resultados: frecuencia de la exposición en los enfermos, frecuencia de la exposición en los no enfermos, frecuencia de la enfermedad o EI en los expuestos, frecuencia de la enfermedad o EI en los no expuestos, frecuencia de los no enfermos y frecuencia de no expuestos.

\section{ESCENARIOS CLÍNICOS Y FR}

Predicción: La presencia de un FR significa un riesgo aumentado de presentar en un futuro una enfermedad o EI, en comparación con sujetos no expuestos. En este sentido son útiles como elementos para predecir la futura presencia de una enfermedad o EI. El riesgo como medida de incidencia expresa la probabilidad que ocurra una enfermedad o EI en una población, en un periodo especificado. Lo adecuado de tal predicción, depende de qué tan parecida es la población en la que se estimó el riesgo, con respecto a la población sobre la cual se hará la inferencia o se extrapolarán los datos.

Causalidad: La presencia de un FR no es necesariamente causal. El aumento de incidencias de un EI entre un grupo expuesto y uno no expuesto, se asume como FR, sin embargo esta asociación puede ser debida a una tercera variable, la que se conoce como variable de confusión.

Diagnóstico: La presencia de un FR aumenta la probabilidad que se presente o identifique un EI. Este conocimiento se utiliza en el proceso diagnóstico ya que las pruebas diagnósticas tienen un valor predictivo positivo más elevado, en sujetos con mayor prevalencia de enfermedad. El conocimiento de los FR se utiliza también para mejorar la eficiencia de los programas de cribaje, mediante la selección de subgrupos de sujetos con mayor riesgo.

Prevención: Si un FR se asocia a un EI, su eliminación reducirá la probabilidad de su presencia. Este es el objetivo de la prevención primaria. Así por ejemplo se asocia la obesidad y la hipertensión, la hipercolesterolemia y la enfermedad coronaria, el tabaco y el cáncer de pulmón, etc. (Pita Fernández, 1995).

\section{APLICACIÓN DE MEDIDAS DE ASOCIACIÓN E IM- PACTO}

Las medidas de asociación son indicadores epidemiológicos que evalúan la fuerza con la que una determinada enfermedad o EI se asocia con un determinado fac- 
tor, que se presume como su causa (MacMahon \& Trichopoulos; Fletcher et al.).

Epidemiológicamente, las medidas de asociación son comparaciones de incidencias: la incidencia del EI en sujetos expuestos al factor estudiado contra la incidencia de la enfermedad o EI en sujetos no expuestos al factor estudiado.

Estadísticamente, las medidas de asociación e impacto cuantifican la relación existente entre variables independientes (de exposición o FR) y dependientes (de efecto, enfermedad o EI). En definitiva lo que estos indicadores miden es la magnitud de la diferencia observada.

Debido a que las medidas de asociación establecen la fuerza con la que la exposición se asocia al EI, bajo ciertas circunstancias estas medidas permiten realizar inferencias causales, especialmente cuando se pueden evaluar mediante una función estadística (Sackett, 1996).

Las medidas de asociación más sólidas se calculan utilizando la incidencia, ya que esta medida de frecuencia permite establecer que el efecto (enfermedad o EI) es posterior a la causa (exposición). En estos casos, se dice, existe una correcta relación temporal entre causa y efecto. Empero, existe un importante porcentaje de estudios en los que no existe suficiente información para calcular la incidencia.

Es así como, existen las denominadas medidas de efecto y las medidas de impacto. Ambas, permiten conocer la magnitud cuantitativa de la fuerza de asociación entre dos variables.

Medidas de efecto: Son aquellas que se basan en el cálculo de un cociente; y permiten se conocen cuantificar discrepancias en la ocurrencia de enfermedad o EI en grupos que difieren en la presencia o no de cierta característica. Estas son: la razón de tasas de incidencia o riesgo absoluto (RA) y el riesgo relativo $(\mathrm{RR})$.

Medidas de impacto: Son aquellas que se basan en las diferencias; y en general, indican la contribución de un determinado factor en la producción de enfermedad o EI entre los que están expuestos a él. Su uso se basa en la suposición de que tal factor es responsable de la aparición del EI y en la presunción de que; de no existir, los riesgos en ambos grupos serían iguales. Por este motivo, se dice que las medidas de impacto indican el riesgo de enfermar que podría evitarse si se eliminara la exposición (Moreno-Altamirano et al.; Fletcher et al.), y están representadas entre otras por: la reducción absoluta de riesgo (RAR), la reducción relativa de riesgo (RRR), el número necesario a tratar (NNT) y el número necesario a dañar (NND) (Replogle \& Johnson; Akobeng).
Entonces, cuando se lee críticamente un artículo en que se comparan grupos, se ha de exigir que los resultados se reporten utilizando este tipo de herramientas, o que al menos, los autores publiquen los datos con los cuales se puedan calcular las medidas de efecto y de impacto antes señaladas; es decir RA, RR, RAR, RRR, NNT y NND (Fletcher et al.; Díaz, 2009; Manterola \& Cortés); para los cuales se requiere generar una tabla de contingencia que simplifica los cálculos (Fig. 2).

\begin{tabular}{|l|c|c|c|}
\cline { 2 - 4 } \multicolumn{1}{c|}{} & Con El & Sin El & Total \\
\hline Expuestos & $\mathrm{a}$ & $\mathrm{b}$ & $\mathrm{a}+\mathrm{b}$ \\
\hline No expuestos & $\mathrm{c}$ & $\mathrm{d}$ & $\mathrm{c}+\mathrm{d}$ \\
\hline Total & $\mathrm{a}+\mathrm{c}$ & $\mathrm{b}+\mathrm{d}$ & $\mathrm{a}+\mathrm{b}+\mathrm{c}+\mathrm{d}$ \\
\hline
\end{tabular}

Fig. 2. Tabla de contingencia necesaria para realizar cálculo de las medidas de asociación.

Riesgo absoluto (RA). El RA o incidencia corresponde a una proporción que se puede definir como el número de sujetos que presentan el EI en un momento dado de tiempo (eventos nuevos) sobre el número de sujetos en riesgo en ese momento. Es decir, es la probabilidad de desarrollar un EI en un determinado periodo de tiempo (Sackett; Díaz). Al calcular la probabilidad de presentar el EI en los expuestos, se obtiene el RA de los expuestos (RA E). Por otra parte, al calcular la probabilidad de presentar el EI en los no-expuestos, se obtiene el RA de los no-expuestos (RA NE) (Figs. $3 \mathrm{~A}$ y $3 \mathrm{~B})$. Se puede expresar como número absoluto o porcentaje. Usualmente se observa que los expuestos tienen mayor probabilidad de enfermar que los no expuestos; sin embargo, esta tesitura no siempre se da ni es suficiente, por lo que se ha de calcular una medida relativa que pondere ambos riesgos basada en la comparación de ambos grupos a través de sus respectivos RA, lo que permite valorar el sentido y la magnitud de una diferencia cuando ella está presente; a esto responde el concepto de RR.

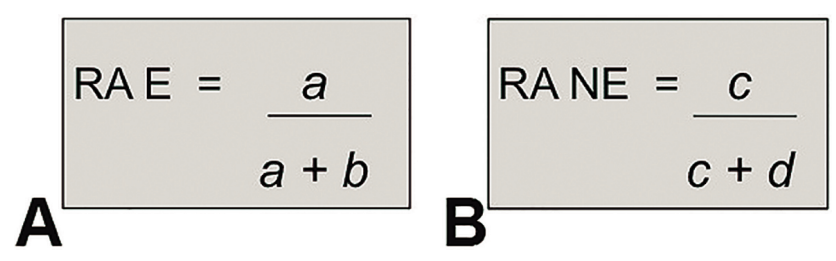

Fig. 3. Fórmulas para el cálculo del RA de expuestos (A) y no expuestos (B).

Riesgo relativo (RR). El RR representa la fuerza de la asociación entre la exposición y la enfermedad o EI. Indica la probabilidad de que se desarrolle el EI en los expues- 
tos a un FR en relación al grupo de los no expuestos. Se calcula dividiendo la incidencia del EI en los expuestos entre la incidencia del EI en los no expuestos (Fig. 4). Cuando el $\mathrm{RR}=1$, significa que la presencia del factor no modifica la probabilidad de ocurrencia del EI. Cuando el RR es > 1, indica cuanto mayor es la probabilidad del EI en los expuestos respecto de los no expuestos (en este caso, se trata de un FR porque aumenta la probabilidad de enfermar). Cuando el $R R$ es < 1 , indica cuanto menor es la probabilidad de enfermar en los expuestos respecto de los no expuestos (se trata entonces de un factor protector porque disminuye la probabilidad de enfermar). Es interesante enfatizar que esta interpretación varía según si la variable resultado sea un evento favorable o desfavorable; es así como si se trata de mortalidad, un RR < 1 indica que la intervención es protectora, en cambio si se trata de supervivencia, un RR > 1 significa que la intervención es perjudicial (Sackett; Díaz).

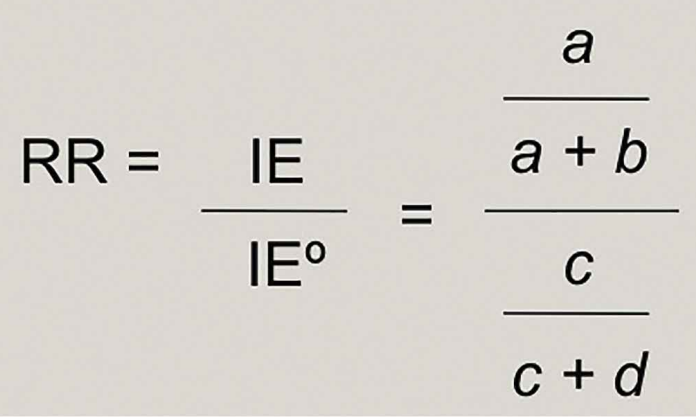

Fig. 4. Fórmula para el cálculo del RR. IE y $\mathrm{IE}^{\circ}$ corresponden a la incidencia en expuestos y no expuestos respectivamente.

Intervalo de confianza del RR. Es el intervalo dentro del cual se encuentra la verdadera magnitud del efecto estudiada y medida mediante el cálculo del RR, pero no determinada con exactitud. Habitualmente se menciona el "intervalo de confianza (IC) del 95\%"; que representa el intervalo en que se encuentra el verdadero valor en estudio, en el 95\% los casos. Dicho de otro modo, constituye el "nivel de certeza", pues es la forma en la que se expresa el nivel de precisión.

Hechos interesantes de verificar cuando se valora el IC del 95\%, son los límites de este y si el intervalo incluye o no el valor 1 . Respecto de los límites, siempre será preferible un intervalo estrecho (por ej. RR de 4,3 con IC 95\% de 1,89 a 5,76) que uno amplio (por ej. RR de 4,3 con IC 95\% de 1,09 a 20,76); se ha de considerar que si IC 95\% incluye el 1 (por ej. RR de 4,3 con IC 95\% de 0,89 a 10,67), esto anula el valor del RR como medida de efecto, en este caso de asociación positiva (la presencia del FR sólo se asocia a mayor ocurrencia del EI) (Blume, \& Peipert, 2003; Faulkner et al., 2008).
Reducción absoluta de riego (RAR). También se le denomina Reducción Atribuible del Riesgo y Riesgo Atribuible. Es una medida útil para expresar la efectividad de un tratamiento o de una intervención. Corresponde a la diferencia entre el riesgo en el grupo sin el FR en estudio y el riesgo en el grupo con el FR en estudio. Dicho de otra forma, expresa la reducción del riesgo de aparición del EI en el grupo de sujetos con la intervención en estudio respecto de los sujetos que no reciben esta intervención, que reciben un placebo o que reciben una intervención diferente. Se calcula entonces como la diferencia del RA o incidencia en el grupo no expuesto y el RA o incidencia en el grupo expuesto (Fig. 5). El valor del RAR puede variar entre -1 y 1; por lo que se debe interpretar de la siguiente forma: si es igual a 0 , significa que no hay asociación entre el FR y el EI; si el valor es menor a 0 , significa que la asociación es positiva, es decir que la presencia del FR se asocia a mayor ocurrencia del EI; y si el valor es mayor a 0 , significa que la asociación es negativa, es decir que la presencia del FR se asocia a menor ocurrencia del EI (Blume \& Peipert; Faulkner et al.; GENESIS, 2014).

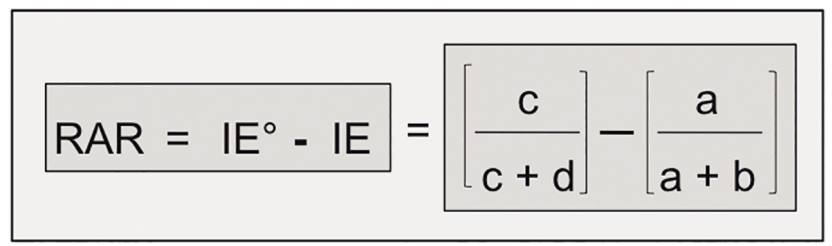

Fig. 5. Fórmula para el cálculo de la RAR. Donde IE y IE ${ }^{\circ}$ corresponden a la incidencia en expuestos y no expuestos respectivamente.

Reducción relativa de riego (RRR). Es otra medida útil para expresar la efectividad de un tratamiento o de una intervención. Se conoce también como fracción atribuible o diferencia relativa de riesgo. Corresponde al cociente entre la RAR y el riesgo del grupo control (no expuesto). Indica así que el riesgo del grupo tratado se reduce un número o porcentaje del riesgo del grupo control (Fig. 6). El principal inconveniente de calcular al RRR es que no permite diferencia los riesgos o beneficios muy grandes de los muy pequeños y no varía de acuerdo al tamaño de la muestra (Blume \& Peipert; Faulkner et al.; GENESIS).

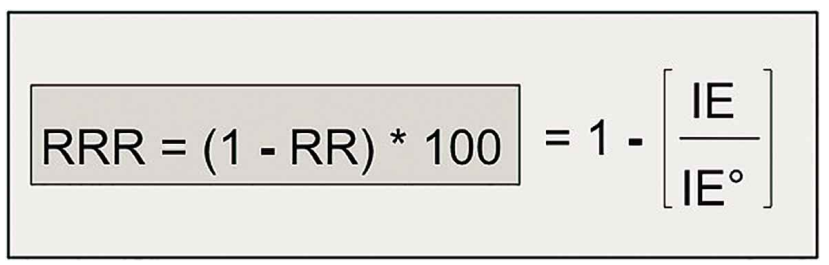

Fig. 6. Fórmula para el cálculo de la RRR. Donde RR corresponde

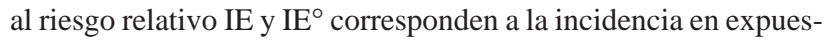
tos y no expuestos respectivamente. 
Número necesario a tratar (NNT). Medida útil para evaluar el impacto de un tratamiento o de una intervención. Se define como el número de individuos que hay que tratar con la terapia experimental para producir, o evitar, un evento adicional respecto a los que se producirían con la terapia estándar o el placebo (Cook \& Sackett, 1995; GENESIS, 2014; ). Permite expresar la magnitud del efecto de un tratamiento en términos comprensibles, lo que facilita la toma de decisiones en salud (Cook \& Sackett, 1995); expresa el efecto del tratamiento en términos que permiten comparar sus ventajas con sus inconvenientes (efectos adversos, costes, etc.). Se calcula como el cociente entre 1 y el RAR (Fig. 7).

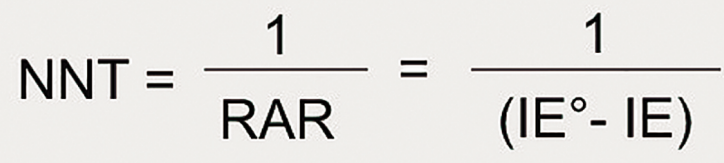

Fig. 7. Fórmula para el cálculo del NNT.

Número necesario a dañar (NND). Este índice se puede usar para evaluar efectos adversos de una intervención. Se puede definir como el número de sujetos que deberían recibir el tratamiento experimental en lugar del estándar o el placebo, para que un sujeto adicional obtenga un perjuicio. Su cálculo tiene sentido cuando el riesgo del evento perjudicial es mayor en el grupo sometido al tratamiento experimental que en el grupo con tratamiento estándar. Representa que el tratamiento experimental consigue menos beneficio que el estándar o un placebo;, o que los efectos adversos inherentes al tratamiento son mayores en el grupo experimental. Existen calculadoras online que permiten obtener fácilmente el NNT y el NND con sus respectivos IC 95\% (Sierra, 2005). El NND se calcula con respecto a la "exposición" y "no exposición", y puede determinarse para los datos brutos o corregidos de factores de confusión; de tal modo que fármacos con NND bajo pueden estar indicados en situaciones en las que el NNT, es menor que el NND. Su fórmula se aprecia en la (Fig. 8).

$$
\text { NND }=\frac{1}{\text { Prop. Event. Adv. grupo NE - Prop. Event. Adv. grupo expuesto }}
$$

Fig. 8. Fórmula para el cálculo del NND.

Índice NNT/NND. Advierte respecto de los efectos beneficioso del fármaco en contraste con los efectos adversos del mismo; del tal forma que una relación menor a 1 , significa que la aplicación con el fármaco en estudio puede ser más beneficioso que perjudicial. Por el contrario si la relación es mayor a 1, se debe considerar de forma rigurosa la eficacia o eficiencia de la intervención terapéutica (Vizcaíno \& Vizcaíno-Carruyo, 2012).

\section{EJEMPLO 1. CÁLCULO E INTERPRETACIÓN DEL RIESGO EN UN ESTUDIO DE COHORTES.}

Para ejemplificar esta materia, utilizaremos un estudio de cohorte de reciente publicación, cuyo objetivo fue determinar si la ruptura intrabiliar (RIB) de un quiste hidatídico del hígado (QHH) es un FR para el desarrollo del EI morbilidad postoperatoria (MPO) (Manterola et al., 2010). Para ello, el primer paso es construir una tabla de contingencia, a partir de la cual se realizarán los cálculos de riesgo (Fig. 9).

\begin{tabular}{|l|c|c|c|}
\cline { 2 - 4 } \multicolumn{1}{c|}{} & MPO (+) & MPO (-) & Total \\
\hline QHH con IBR & 34 & 122 & 156 \\
\hline QHH sin IBR & 9 & 87 & 96 \\
\hline Total & 43 & 209 & 252 \\
\hline
\end{tabular}

Fig. 9. Tabla de contingencia necesaria para realizar cálculo de las medidas de asociación del ejemplo 1 (Manterola et al., 2010).

De este modo, y de acuerdo con los datos del ejemplo, el RA de desarrollar MPO en los sujetos con IBR es de 21,8 ; y en quienes no tienen IBR es de 9,4. Estos datos nos permiten determinar que el RR es de 2,32 (IC 95\% de 1,17 $14,63)$; lo que significa que la probabilidad de desarrollar MPO en el grupo de individuos con RIB es 2,3 veces mayor que en el grupo de pacientes sin RIB. Esto permite concluir que la existencia de IBR en sujetos con QHH es un FR para el desarrollo de MPO.

La estimación de RRR en este caso no aplica porque el riesgo en el grupo expuesto es mayor que el riego del grupo no expuesto. Por otra parte, la RAR da un valor de 12,4 , lo que representa una asociación positiva, es decir, que la presencia de IBR se asocia a una mayor ocurrencia de desarrollo de MPO en sujetos con QHH.

Por otra parte, como en este estudio no se evalúan tratamientos, porque corresponde a un escenario de pronóstico, no aplica el cálculo de NNT ni de NND.

\section{EJEMPLO 2. CÁLCULO E INTERPRETACIÓN DEL RIESGO EN UN ENSAYO CLÍNICO.}

Para ejemplificar esta materia, utilizaremos un ensayo clínico publicado por Laterre et al. (2004), cuyo objetivo 
fue comparar las diferencias en la mortalidad hospitalaria y recursos utilizados de en adultos graves por sepsis que fueron aleatorizados para recibir drotrecogina alfa activado (DAA) o placebo en el ensayo PROWESS ( $\mathrm{n}=850$ vs. $\mathrm{n}=$ 840). Para ello, el primer paso es construir una tabla de contingencia, a partir de la cual se realizarán los cálculos de riesgo (Fig. 10).

\begin{tabular}{|l|c|c|c|}
\cline { 2 - 4 } \multicolumn{1}{c|}{} & Muerte & No muerte & Total \\
\hline DDA & 210 & 640 & 850 \\
\hline Placebo & 259 & 581 & 840 \\
\hline Total & 469 & 1221 & 1690 \\
\hline
\end{tabular}

Fig. 10. Tabla de contingencia necesaria para realizar cálculo de las medidas de asociación del ejemplo 2 (Laterre et al., 2004).

De este modo, y de acuerdo con los datos del ejemplo, el RA expuestos a DDA fue de 0,247 y el RA de los no expuestos a DDA fue de 0,308 (la incidencia de muerte en los expuestos a DDA es menor que la de aquellos con placebo).

La RAR es de 0,061 , lo que representa que el tratamiento con DDA reduce el riesgo de morir en un $6,1 \%$.
El RR es de 0,8019, lo que implica que la intervención con DDA, tiene un efecto protector respecto al evento que se mide.

La RRR es de 0,198, lo que implica que el tratamiento con DDA reduce el riesgo de muerte respecto del grupo control.

El NNT es de 16,4; lo que significa que ese es el número de pacientes que deben recibir tratamiento con DDA para evitar una muerte.

\section{CONCLUSIONES}

Dadas las actuales condiciones, en cuanto a proliferación y disponibilidad de publicaciones científicas, al mismo tiempo que se hace necesario conocer los parámetros para reparar en la validez de estos estudios, los clínicos que observan la evidencia para una mejor atención de sus pacientes, deberían además familiarizarse con las distintas formas de presentación de los resultados e interpretarlos no sólo a la luz de su significación estadística.

Por lo mismo, derivado de un correcto reconocimiento de las distintas medidas de asociación y la adecuada interpretación de los resultados de las mismas, éstas podrían ser útiles y considerarse para ser aplicadas en nuestra práctica diaria.

MANTEROLA, D. C. \& OTZEN, H. T. Clinical risk assessment, interpretation and practical utility. Int. J. Morphol., 33(3):842-849, 2015 .

SUMMARY: Clinicians who observed the evidence for better care of their patients, should be familiar not only with some tools of critical literature reading of biomedical articles, but also with some epidemiological concepts and statistical tools that are useful to assess the validity of these studies. We are referring particularly to the measures of association and impact which permit us the ability to summarize statistical information from the articles concerned, as a summary of data and analysis and interpretation of results with both statistical and clinical significance. We understand that this is not easy because these are dry concepts, and furthermore, it is rare that health staff have incorporated probabilistic reasoning, and because ultimately, the way these results are reported is not the most didactic and practical. However, given the increasing proliferation and availability of scientific publications, it becomes more and more essential to understand these concepts and their usefulness in clinical practice. Therefore, the aim of this paper is to describe the theoretical concepts for understanding the importance of risk analysis, to know the method of calculation of the main measures of association and impact used in clinical research, as well as its interpretation and practical application in different types of study designs.

KEY WORDS: Risk; Incidence; Cohort studies; Clinical trial; Epidemiology; Evidence-Based Medicine.

\section{REFERENCIAS BIBLIOGRÁFICAS}

Abraira, V. Medidas del efecto de un tratamiento (I): reducción absoluta del riesgo, reducción relativa del riesgo y riesgo relativo. Semergen,26(11):535-6, 2000.
Akobeng, A. K. Understanding measures of treatment effect in clinical trials. Arch. Dis. Child., 90(1):54-6, 2005. 
Blume, J. \& Peipert, J. F. What your statistician never told you about P-values. J. Am. Assoc. Gynecol. Laparosc., 10(4):43944, 2003

Cook, R. J. \& Sackett, D. L. The number needed to treat: a clinically useful measure of treatment effect. B. M. J., 310(6977):452-4, 1995.

Díaz, V. P. Metodología de la investigación científica y bioestadística para profesionales y estudiantes de ciencias de la salud. 2a ed. Santiago, RIL Editores, 2009.

Fahey, T.; Griffiths, S. \& Peters, T. J. Evidence based purchasing: understanding results of clinical trials and systematic reviews. B. M. J.,311(7012):1056-9, 1995.

Faulkner, C.; Fidler, F. \& Cumming, G. The value of RCT evidence depends on the quality of statistical analysis. Behav. Res. Ther., 46(2):270-81, 2008.

Fletcher, R. H.; Fletcher, S. W. \& Wagner, E. H. Epidemiología clínica. Aspectos fundamentales. $2^{\mathrm{a}}$ ed. Barcelona, Masson, 2003.

Grupo de Evaluación de Novedades, Estandarización e Investigación en Selección de medicamentos (GENESIS). Enlaces de interés. Calculadoras de NNT, RR y RRA con intervalos de confianza $95 \%$, en Internet. Variables binarias. Madrid, Sociedad Española de Farmacia Hospitalaria, 2014. Disponible en: http://gruposdetrabajo.sefh.es/genesis/genesis/Enlaces/ Calculadoras.htm

Laterre, P. F.; Levy, H.; Clermont, G.; Ball, D. E.; Garg, R.; Nelson, D. R.; Dhainaut, J. F. \& Angus, D. C. Hospital mortality and resource use in subgroups of the Recombinant Human Activated Protein C Worldwide Evaluation in Severe Sepsis (PROWESS) trial. Crit. Care Med., 32(11):2207-18, 2004.

MacMahon, B. \& Trichopoulos, D. Epidemiology: Principles and Methods. 2nd ed. Boston, Little Brown \& Company, 1996.

Manterola D., C. \& Cortés J., M. Cómo valorar e interpretar un artículo sobre tratamiento o procedimientos terapéuticos. Rev. Chil. Cir., 62(6):639-48, 2010.

Manterola, C.; Vial, M.; Sanhueza, A. \& Contreras, J. Intrabiliary rupture of hepatic echinococcosis, a risk factor for developing postoperative morbidity: a cohort study. World J. Surg., 34(3):581-6, 2010.

Manterola, C. \& Otzen, T. Estudios observacionales. Los diseños utilizados con mayor frecuencia en investigación clínica. Int. J. Morphol.,32(2):634-45, 2014.

Moreno-Altamirano, A.; Lopez-Moreno, S. \& Corcho-Berdugo, A. Principales medidas en epidemiología. Salud Pública Méx., 42(4):337-48, 2000
Palmeiro Fernández, G.; Casado Górriz, I.; Vázquez Fernández, L. A. \& Díaz Grávalos, G. J. Impact of the format of presentation of results in clinical trials on the intention of prescription in primary care. A crossed clinical trial in Ourense. Rev. Esp. Salud Publica, 78(1):27-40, 2004.

Pineda Ovalle, L. F.; Sierra Arango, F. \& Otero Regino, W. Interpretación y utilidad de las principales medidas en epidemiología clínica. Rev. Col. Gastroenterol., 21(3):198-206, 2006.

Pita Fernández, S.; Vila Alonso, M. T. \& Carpente Montero, J. Determinación de factores de riesgo. Fisterra.com Atención Primaria en la Red, Elsevier, 2002. Disponible en: https:// www.fisterra.com/mbe/investiga/3f_de_riesgo/ 3f_de_riesgo2.pdf

Pita Fernández, S. Epidemiología. Conceptos básicos. En: Tratado de Epidemiología Clínica. Madrid, DuPont Pharma, 1995. pp.25-47.

Replogle, W. H. \& Johnson, W. D. Interpretation of absolute measures of disease risk in comparative research. Fam. Med., 39(6):432-5, 2007.

Sackett, D. L. On some clinically useful measures of the effects of treatment. Evid. Based Med., 1:37-8. 1996.

Sierra, F. Evidence-Based Medicine (EBM) in practice: applying number needed to treat and number needed to harm. Am. J. Gastroenterol., 100(8):1661-3, 2005.

Vizcaíno, G. \& Vizcaíno-Carruyo, J. El número necesario a tratar como medida de efecto en el tratamiento de la trombocitopenia inmune primaria. Invest. Clin. (Maracaibo), 53(1):16-27, 2012.

\author{
Dirección para Correspondencia: \\ Dr. Carlos Manterola \\ Departamento de Cirugía y Traumatología \\ Universidad de La Frontera \\ Casilla 54-D \\ Temuco \\ CHILE
}

Email: carlos.manterola@ufrontera.cl

Recibido : 27-03-2015

Aceptado: 19-06-2015 